\title{
Coexistence Mechanisms for Interference Mitigation in the 2.4-GHz ISM Band
}

\author{
Carla-Fabiana Chiasserini, Member, IEEE, and Ramesh R. Rao, Senior Member, IEEE
}

\begin{abstract}
Wireless technologies sharing the same frequency band and operating in the same environment often interfere with each other, causing severe decrease in performance. In this paper, we propose two coexistence mechanisms based on traffic scheduling techniques that mitigate interference between different wireless systems operating in the 2.4-GHz industrial, medical, and scientific band. In particular, we consider IEEE 802.11 wireless local area networks (WLANs) and Bluetooth (BT) voice and data nodes, showing that the proposed algorithms can work when the two systems are able to exchange information as well as when they operate independently of one another. Results indicate that the proposed algorithms remarkably mitigate the interference between the IEEE 802.11 and BT technologies at the expense of a small additional delay in the data transfer. It is also shown that the impact of the interference generated by microwave ovens on the IEEE 802.11 WLAN's performance can be significantly reduced through the mechanisms presented.
\end{abstract}

Index Terms-Coexistence mechanisms, quality of service, wireless local area networks (WLANs), wireless personal area networks (WPANs).

\section{INTRODUCTION}

$\mathbf{T}$ HE NEXT few years will likely bring pervasive deployment of smart wireless devices. To make this vision a reality, devices must be able to share the same frequency band and move between different wireless systems without the need of any licensing procedure [1]. Although the use of unlicensed bands facilitates spectrum sharing and allows for an open access to the wireless medium, it also raises serious challenges, such as mutual interference between different radio systems and spectrum utilization inefficiency.

In this paper, we deal with the problem of mutual interference between two emerging wireless technologies: wireless local area networks (WLANs) and wireless personal area networks (WPANs). In particular, we consider IEEE 802.11 WLANs [2], [3] and short-range radio systems based on the Bluetooth (BT) specification [4], [5] or, equivalently, IEEE 802.15 WPANs [6]. These systems will operate in the $2.4-\mathrm{GHz}$ industrial, scientific, and medical (ISM) frequency band, i.e., the unlicensed spectrum. BT uses a frequency-hopping

Manuscript received August 10, 2001; revised March 4, 2002 and June 1, 2002; accepted June 3, 2002. The editor coordinating the review of this paper and approving it for publication is V. C. M. Leung. This work was supported by the Centro di Eccellenza per le Radio Comunicazioni Multimediali (CERCOM), Torino, Italy.

C.-F. Chiasserini is with the Dipartimento di Elettronica, Politecnico di Torino, 10129 Torino, Italy (e-mail: chiasserini@polito.it).

R. R. Rao is with the Electrical and Computer Engineering Department, University of California, San Diego, CA 92093-0407 USA (e-mail: rao@cwc. ucsd.edu).

Digital Object Identifier 10.1109/TWC.2003.817417 spread spectrum (FHSS) scheme while IEEE 802.11 can either use an FHSS or a direct-sequence spread spectrum (DSSS) technique. WLANs and WPANs are complementary rather than competing technologies, and many application models have been envisioned for situations requiring BT and 802.11 to operate simultaneously and in close proximity [7]. Under these conditions, interference between 802.11 and BT occurs whenever the interference energy is sufficient to cause a decrease of the signal-to-interference ratio at the receiver and the two system transmissions overlap both in frequency and in time.

According to the IEEE 802.15 working group, interference between 802.11 and BT causes a severe degradation of the systems' throughput when the distance between interfering devices is less than $2 \mathrm{~m}$. A slightly less significant degradation is observed when the distance ranges between 2 and $4 \mathrm{~m}$ [8]. In order to mitigate this effect, the IEEE 802.15 working group has created the Task Group 2 (TG2), which is devoted to the development of coexistence mechanisms [6], i.e., techniques that allow 802.11 and BT to operate in a shared environment without significantly impacting the performance of each other [9]. Two classes of coexistence mechanisms have been defined: collaborative and noncollaborative techniques [6]. With collaborative techniques, it is possible for the BT network and the WLAN to exchange information and reduce the mutual interference; however, they can be implemented only when the BT and the 802.11 devices are collocated in the same terminal. With noncollaborative techniques, there is no way to exchange information between the two systems, and they operate independently.

In this paper, we propose two novel coexistence mechanisms, called overlap avoidance (OLA) schemes, which are based on simple traffic scheduling techniques. The first mechanism is to be performed at the IEEE 802.11 in the presence of a BT voice link, the second mechanism at the BT system in the case of a BT data link. The proposed algorithms have the following advantages: 1) they do not need a centralized traffic scheduler; 2) they can be implemented in collaborative or noncollaborative mode; 3 ) they are able to mitigate interference between collocated and noncollocated BT and IEEE 802.11 devices; and 4) they have a minor impact on the IEEE 802.11 standard and on the BT specification. Both schemes are based on the assumption that 802.11 and BT can detect interference due to other technologies sharing the same environment. This assumption is trivially true in a collaborative setting, where information related to traffic transmissions can be directly exchanged between the interfering systems. In a noncollaborative setting, this information can be acquired through channel sensing and assessment of the received signal strength and packet loss rate. This is further discussed in Section IV. 
By applying the OLA mechanisms, a significant reduction in the interference between IEEE 802.11 and BT can be achieved. Similar reductions are expected when other interference sources are considered. Using the OLA scheme at the 802.11 stations reduces the impact on the WLAN performance of any interfering transmission with a periodic pattern. Likewise, the OLA scheme performed by the BT devices is able to counteract the interference generated by any wireless system using a limited frequency span in the ISM band. To illustrate this, we show how the proposed mechanisms improve performance of an 802.11 WLAN suffering the interference caused by microwave ovens.

The remainder of the paper is organized as follows. In Section II, we review previous work on coexistence mechanisms between IEEE 802.11 and BT. Section III briefly describes IEEE 802.11 and BT technology, and introduces the model adopted to evaluate the mutual interference between the two systems. Section IV presents the proposed coexistence techniques. Section V describes the considered simulation scenario. Section VI shows the improvement obtained in the 802.11 and BT performance. The ability of the proposed schemes to cope with the interference generated by microwave ovens is studied in Section VII. Finally, Section VIII concludes the paper.

\section{RELATED WORK}

Several coexistence mechanisms have been proposed within the IEEE 802.15 TG2. As mentioned above, they can be classified as collaborative or noncollaborative mechanisms [10]. There are two important examples of collaborative coexistence mechanisms: the so-called medium access control (MAC) enhanced temporal algorithm (META) [11] and alternating wireless medium access (AWMA) [12], [13] schemes.

In the META technique, an 802.11 station and a BT node are collocated in the same physical unit. META involves the use of a centralized controller that monitors the BT and the 802.11 traffic, allowing the exchange of information between the two collocated radio systems. The controller works at the MAC layer; it provides per-packet authorization of all transmissions and uses its knowledge of the 802.11 and BT activity to predict collisions. When a collision might occur, META schedules transmissions based on simple rules determined by the packet types. In particular, 802.11 acknowledgment packets have higher priority than any BT packets, while BT synchronous connection oriented (SCO) traffic has higher priority than any 802.11 data packets.

The AWMA mechanism operates at the MAC layer and is based on the time-division multiple access scheme. It assumes that the 802.11 access point and the BT master are collocated in the same physical unit [12], [13], and that the 802.11 and BT devices transmit alternately to avoid overlap in time between their transmissions. The 802.11 access point sends out a beacon at a periodic interval and each beacon-to-beacon interval is divided into two subintervals: one for 802.11 traffic and one for BT traffic. AWMA cannot be applied in the case of BT SCO links [13].

Note that both the META and the AWMA schemes are unable to mitigate interference coming from noncollocated devices. Also, since they completely orthogonalize transmissions of technologies sharing the same radio spectrum, the systems' throughput will be significantly decreased as the number of wireless technologies operating in the unlicensed bands grows. The two MAC layer techniques can be integrated with a collaborative physical layer solution, the so-called deterministic frequency nulling scheme [14]. The key idea is that because the BT signal can be considered a narrowband interferer for the 802.11 DSSS signal, we can put a null in the 802.11 receiver at the frequency of the BT signal. Since the BT signal hops in frequency, the 802.11 receiver must know the frequency hopping pattern, as well as the timing, of the BT transmitter. This again implies that the 802.11 and the BT devices must be collocated in the same physical unit.

Noncollaborative coexistence mechanisms include the adaptive frequency hopping (AFH) technique, the adaptive packet selection and scheduling, and the transmit power control/rate scaling scheme.

According to the AFH scheme [15]-[17], BT frequency channels are classified as "good" or "bad" and are used intelligently to reduce the probability of overlap in frequency with the 802.11 signal. Due to FCC restrictions (see Title 47, Part 15.247 (a) [27]), two methods have been defined. In the first method, the so-called Mode $L$, bad channels are removed from the hopping sequence; in the second one, the so-called Mode $H$, some grouping of the bad and good channels is performed so that the hopping sequence can intelligently schedule the use of the bad channels and maximize the use of the good channels.

Adaptive packet selection and scheduling [18] can be effectively used to mitigate interference between 802.11 and BT. By selecting the best BT packet type according to the condition of the upcoming frequency hop, BT throughput is improved. Also, BT transmissions can be scheduled in such a way that hops in the 802.11 band are avoided, thus, reducing interference between the two radio systems.

In [19], the transmit power control/rate scaling scheme is presented. This technique is based on the idea that 802.11 and BT devices should reduce their transmission power as much as possible to obtain satisfactory performance. All 802.11 devices currently implement a rate shifting/control algorithm based on the perceived level of signal quality. The scheme presented in [19] extends this control algorithm to incorporate the highest mandatory rate at lower transmit power, i.e., when possible, the 802.11 devices would shift to the highest rate using lower transmit power.

\section{SYSTEM BACKGROUND}

IEEE 802.11 WLANs cover a range of approximately $100 \mathrm{~m}$ and can operate at bit rates as high as $11 \mathrm{Mb} / \mathrm{s}$. We focus on systems that use the DSSS scheme and consider their bandwidth to be roughly equal to $22 \mathrm{MHz}$ [2], [20]. The fundamental building block of the network is the so-called basic service set (BSS), which is composed of several wireless stations using the same spreading sequence and MAC function. Wireless stations can directly communicate with each other forming an ad hoc network or through a centralized access point that also provides a connection to the wired network [2]. The two fundamental 


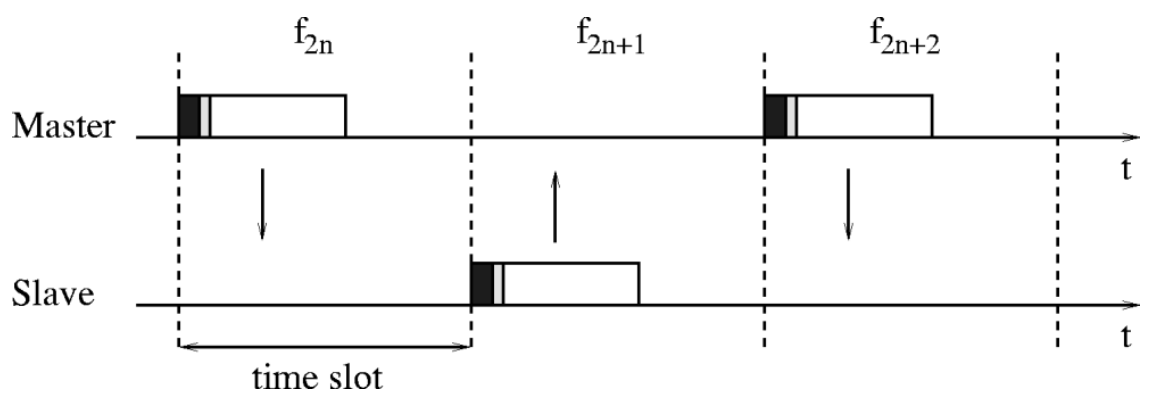

Fig. 1. FH/TDD channel in BT.

MAC schemes defined in the IEEE 802.11 standard are the distributed coordination function (DCF) and the point coordination function. The former is based on the carrier-sense multiple access with collision avoidance protocol and allows for an asynchronous data transport; the latter is based on polling controlled by the access point and is able to support real-time traffic [3]. In this paper, only the DCF scheme is considered.

BT provides interconnection of devices in the user's vicinity; its typical use is in a range of roughly $10 \mathrm{~m}$. The basic architectural unit in BT systems is the piconet, composed of a master device and seven active slave devices at most, which are allowed to communicate with the master only [4], [5]. BT can support up to three SCO links for real-time services, such as voice traffic, and asynchronous connection-less (ACL) links for non real-time applications, such as data traffic. The maximum throughput that can be provided is equal to $721 \mathrm{~Kb} / \mathrm{s}$. An FHSS scheme is used at the physical level with hop rate equal to 1600 hops/s; each master chooses a different hopping sequence so that piconets can operate in the same area without interfering with each other. Hopping frequencies range over 79 frequency channels in the ISM band, each of the channels being $1 \mathrm{MHz}$ wide. The nominal hop dwell time is equal to $625 \mu \mathrm{s}$. A time-division duplex (TDD) technique is used to transmit and receive data in a piconet. Each packet transmitted in a slot occupies $366 \mu$ s; slots are centrally allocated by the master and alternately used for master and slave transmissions. Master transmissions always begin at even slots (namely, in slots $2 n$ with $n=1,2, \ldots$ ), slave transmissions at odd slots (namely, in slots $2 n+1$ with $n=1,2, \ldots$ ). Fig. 1 shows the FH/TDD channel. The BT specification also allows for multislot data transmissions, i.e., for packets occupying more than one slot (namely, three or five slots). In this case, packets are sent by using a single frequency hop, which is the hop corresponding to the slot at which the packet started.

In order to define mechanisms for the coexistence of IEEE 802.11 and BT devices operating in a common area, it is imperative to develop an appropriate model for their mutual interference.

\section{A. Interference Model}

Interference between IEEE 802.11 and BT arises whenever the interfering power from a BT (802.11) transmitter causes a significant decrease in the carrier to interference power margin at the 802.11 (BT) receiver [21]-[25]. By using the method presented in [23]-[25], the number of interfering devices and the associated carrier to interference power margin can be derived from the following system parameters: 1) distance between transmitters and receivers; 2) average density of the transmitters in the considered spatial area; 3) transmission power of the interfering systems; and 4) signal attenuation factor due to propagation.

In this work, we assume that the set of BT devices having sufficient power to cause interference to 802.11 is given, as well as the set of 802.11 stations that cause interference to BT. We also consider that whenever transmissions by these BT and 802.11 devices overlap both in time and in frequency, the signals involved in the collision are received in error with probability equal to one. The average number of symbols "hit" because of a collision between 802.11 and BT can be, therefore, computed as follows.

We denote the BT time slot with $T_{\mathrm{BI}}$, the actual BT transmission time per slot with $T_{\mathrm{BP}}$, and the 802.11 packet time duration with $T_{W}$. Let $x$ be the time period from the beginning of the first overlapping BT slot to the beginning of the 802.11 packet [ $x$ ranges in the time interval $\left.\left(0, T_{\mathrm{BI}}\right)\right]$. The number of BT slots that overlap the 802.11 packet in time depends on $x$ and can be derived as [22]

$$
N(x)= \begin{cases}\left\lceil\frac{T_{W}}{T_{\mathrm{BI}}}\right\rceil, & \text { if } x \leq T_{\mathrm{BI}} \cdot\left\lceil\frac{T_{W}}{T_{\mathrm{BI}}}\right\rceil-T_{W} \\ \left\lceil\frac{T_{W}}{T_{\mathrm{BI}}}\right\rceil+1, & \text { else. }\end{cases}
$$

Fig. 2 shows an example with $N(x)=5$ and packet length equal to one slot. Variables $T_{i}(i=1, \ldots, N(x))$ indicate the portion of the $i$ th BT slot that actually interferes with the 802.11 packet. For the generic time slot $i(i=1, \ldots, N(x))$, we have that $T_{i}=0$ if no BT transmission occurs in interval $i$; otherwise as shown in (2) at the bottom of the page [22]. By fixing the

$$
T_{i}= \begin{cases}\max \left(T_{\mathrm{BP}}-x, 0\right), & i=1 \\ T_{\mathrm{BP}}, & i=2, \ldots, N(x)-1 \\ \min \left(x+T_{W}-(N(x)-1) T_{\mathrm{BI}}, T_{\mathrm{BP}}\right), & i=N(x)\end{cases}
$$




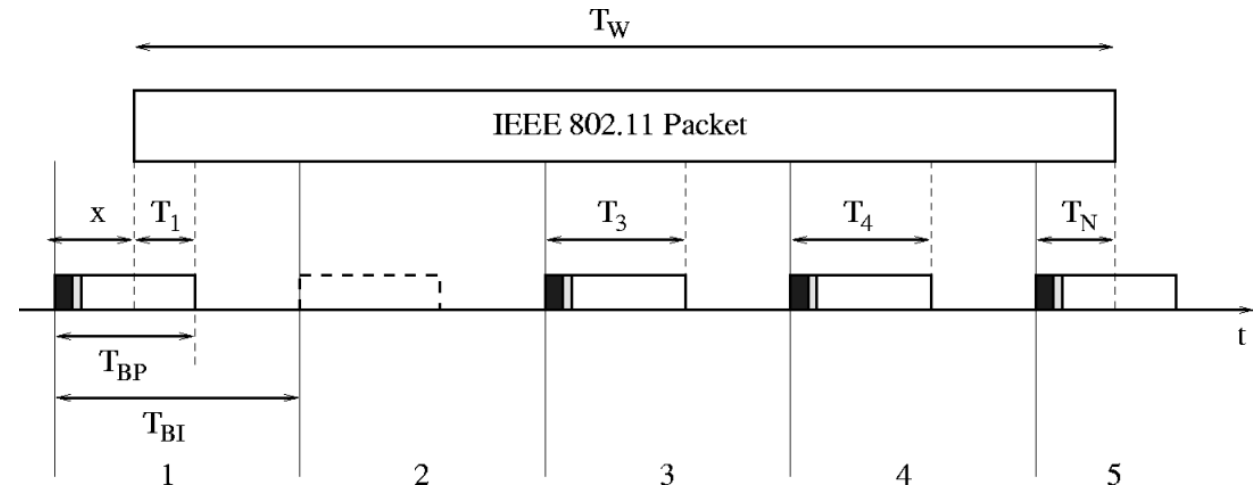

Fig. 2. Overlap between IEEE 802.11 and BT packets.

value of $x$ for $i=1, \ldots, N(x)$, we define $\delta_{i}$ as the probability that BT traffic is transmitted in slot $i(i=1, \ldots, N(x))$,

$$
\delta_{i}= \begin{cases}0, & \text { if the } i \text { th BT slot is idle } \\ 1, & \text { otherwise }\end{cases}
$$

By considering that the 802.11 stations use a DSSS scheme and neglecting out-of-band interference, the probability that BT and 802.11 overlap in frequency is equal to the probability that BT hops on the 22-MHz WLAN DSSS band. From the procedure used to generate the BT hopping sequences [5], it follows that the BT hopping on the WLAN band can be approximated by an independent identically distributed process with parameter $h_{f}$. When no coexistence mechanism is applied, we can write [26]: $h_{f}=(22 / 79)=0.278$, where 22 and $79 \mathrm{MHz}$ are the 802.11 and the BT bandwidth, respectively.

The average number of symbols "hit" because of a collision between BT and 802.11 can, therefore, be written as

$$
\eta_{x}=h_{f}\left(T_{1}^{(s)} \delta_{1}+\sum_{i=2}^{N(x)-1} T_{i}^{(s)} \delta_{i}+T_{N(x)}^{(s)} \delta_{N(x)}\right)
$$

where we denote with $T_{i}^{(s)}$ the ratio $T_{i} / T_{s}(i=1, \ldots, N(x))$, with $T_{s}$ being the symbol time duration. From (4), it is clear that in order to mitigate the mutual interference between BT and 802.11, we need to make either $N(x), h_{f}$ or $\delta_{i}(i=1, \ldots, N(x))$ small. A small $N(x)$ can be obtained by using short WLAN packets, which, however, increases the 802.11 transmission overhead. A small $h_{f}$ requires reducing the probability that 802.11 and BT transmissions overlap in frequency, while a small $\delta_{i}$ implies a low probability of overlap in time between the two systems transmission.

\section{OLA COEXISTENCE MEChANISMS}

Based on the previous findings, we develop two coexistence algorithms, named OLA mechanisms that use simple traffic scheduling techniques at the MAC layer.

The first algorithm, denoted by voice-OLA (V-OLA), is used in the case of BT SCO links. This scheme avoids overlap in time between the BT SCO traffic and the 802.11 packets by performing a proper scheduling of the traffic transmissions at the WLAN stations. In a BT network, each SCO link occupies FH/TDD channel slots according to a deterministic pattern.
Thus, an 802.11 station shall start transmitting when the BT channel is idle and adjust the length of the WLAN packet so that it fits between two successive BT transmissions. The second algorithm, denoted by data-OLA (D-OLA), is suitable for BT data links. As described in Section III, the length of the BT packets can be equal to one, three, or five time slots. In the case of multislot transmissions, packets are sent by using a single frequency hop, which is the hop corresponding to the slot at which the packet started. The key idea of the D-OLA algorithm, described in more detail below, is to use the variety of packet lengths that characterize the BT system to avoid overlap in frequency between 802.11 and BT transmissions. Within each interfering piconet, the D-OLA algorithm induces the BT master device to schedule data packets with the proper duration (i.e., one, three, or five slots) in order to skip the frequency locations of the hopping sequence that are expected to drop on the 802.11 band. The two proposed mechanisms are jointly applied when both SCO and ACL links are active over the BT channel.

The proposed schemes are based on the assumption that both 802.11 and BT devices can detect interference due to other technologies sharing the same environment and using the same frequency band. This assumption is trivially true in a collaborative setting where BT and 802.11 can directly exchange information related to their traffic transmissions. In a noncollaborative setting, this information can be acquired through channel sensing and assessment of the received signal strength and of the packet loss rate. This issue is further discussed below for each of the proposed schemes.

\section{A. V-OLA Mechanism}

In the case of BT SCO traffic, slots are allocated according to a deterministic pattern; for instance, for each SCO connection using a HV3-type link [5], a single-slot packet is transmitted periodically in both directions every six time slots. Whenever a BT packet hops in the 802.11 frequency band, an 802.11 station in receive mode ${ }^{1}$ senses the $\mathrm{BT}$ transmission as colored noise, i.e., as a signal with a specific behavior in time and in frequency. In a noncollaborative setting, an 802.11 station can detect the time intervals that are occupied by interfering transmissions by using the clear channel assessment (CCA) procedure. In particular, the primitive PMD_ED.INDICATE allows an 802.11 device

1802.11 and BT are half-duplex systems (i.e., devices cannot simultaneously transmit and receive). 
to detect any radio frequency signal, that is above a predefined threshold. More details on the primitives used by 802.11 stations to detect interference on the radio channel can be found in [3, pp. 214-215].

If SCO and ACL links are simultaneously active on the BT channel, the D-OLA scheme is also applied and, as explained later, the probability that an ACL packet hops on the 802.11 band becomes negligible. This implies that an 802.11 station is likely to detect interference due to the BT SCO traffic only. Due to the periodicity and the predefined time duration of the BT SCO packets, the 802.11 device can estimate the BT signal pattern. However, two problems still need to be solved. First, the BT signal hops in the 802.11 band with probability equal to $h_{f}$. Thus, even if an 802.11 station can start applying the V-OLA mechanism as soon as the BT interference is sensed, it can hardly detect the time instant when the interference due to the BT SCO transmission ends. We assume that an 802.11 station considers the BT SCO transmission terminated if it does not detect the interference signal for a time interval equal to $10 \mathrm{~ms}$ (which corresponds to $16 \mathrm{BT}$ time slots). Second, once the BT interference is detected, the timing of the BT packet transmission may drift, thus, resulting in an inaccurate prediction of the BT signal by the 802.11 device. Although this is an interesting subject for future research, we neglect the effect of the timing drift of the BT transmissions on the performance of the V-OLA scheme.

Whenever an 802.11 station is ready to transmit, it acts according to the information acquired on the pattern of the BT SCO traffic. If the channel is idle and no interference is expected for a time period equal to the next $(i-1)$ BT slot duration, the 802.11 station transmits a data packet with payload size equal to the minimum of $(i \cdot 500)$ and $1500 \mathrm{~B}$. The minimum payload has been set to $500 \mathrm{~B}$ to make the corresponding 802.11 packet transmission time comparable to the duration of a single-slot BT packet. Conversely, if the channel is occupied by an interfering signal, the WLAN station can either: 1) send a packet with a 500-B payload (shortened transmission (ST) mode) or 2) refrain from transmitting (postponed transmission (PT) mode).

With the ST mode, the 802.11 transmission does not necessarily overlap in time with the BT packets because a one-slot BT packet lasts only slightly longer than half the duration of one time slot. Besides, even in the case of time overlap, 802.11 and BT packets collide only if BT packets hop on the WLAN frequency band.

When a WLAN station refrains from transmitting, i.e., it acts in PT mode, the 802.11 transmission is postponed by computing a new backoff time. In this case, two opposite effects take place: on the one hand, a lower overlap probability is achieved than in the case where a short packet is transmitted; on the other hand, the WLAN stations' access delay increases and the WLAN channel utilization decreases with respect to the case where the ST mode is applied.

\section{B. D-OLA Mechanism}

We consider a BT data link and assume that the BT master devices are aware of which frequency channels are occupied by the interfering 802.11 stations. An 802.11 system does not typically move from its $22-\mathrm{MHz}$ frequency band. Therefore, in a noncollaborative setting, a BT device can identify the frequency channels that are occupied by the WLAN by using any of the following methods [17].

1) BT devices gradually determine which channels are occupied based on the observed packet loss.

2) BT devices assess the received signal strength (RSSI) across the radio environment before they start operating.

3) BT devices transmit "test" packets across the frequency spectrum, observe the packet loss rate over the channels, and discover the band used by an interfering system.

Notice that Methods 1 and 2 are already included in the BT specification; while, POLL-NULL messages, which are exchanged between master and slave, can be used as "test" packets to implement Method 3 [17].

Let us focus on the FH/TDD channel of one BT piconet. Recall that a master transmission always begins in even slots while slaves can start transmitting in odd slots only. For the sake of simplicity, we assume that default data packets are one-slot long. Let us denote by $f_{m}$ the frequency location of the hopping sequence at the generic time slot $m$ and let the current time slot be equal to $2 n$.

Consider first that following $f_{2 n}, f_{2 n+1}$ hops on the 802.11 band. Notice that $f_{2 n}$ and $f_{2 n+1}$ shall correspond to a master and a slave transmission, respectively. According to the D-OLA algorithm, if enough data are buffered at the master for the intended slave, the master schedules a multislot packet instead of a single-slot packet. In this way, frequency hop $f_{2 n+1}$ is skipped; for instance, if a three-slot packet is sent, the next slave transmission will use $f_{2 n+3}$. If too little data is available, the master acts by default and sends a single-slot packet.

Next, assume that among the frequency locations following $f_{2 n}, f_{2 n+2}$ hops on the 802.11 band. Notice that frequency location $f_{2 n+2}$ corresponds to a master transmission. In this case, at time slot $2 n$, the master asks the slave, transmitting in the next slot, to send a multislot packet so that $f_{2 n+2}$ is skipped. If the slave has enough data to send, for example, a three-slot packet, the slave transmission extends from slot $2 n+1$ to slot $2 n+3$ by using frequency $f_{2 n+1}$ only. The next slot allocated for the master transmission will, therefore, hop on frequency location $f_{2 n+4}$. A similar mechanism is applied when default data transmissions use three- or five-slot packets.

The scheduling algorithm could also prevents the master (slave) from transmitting in the time slot corresponding to a frequency that hops on the 802.11 band whenever there is not enough data in the buffer at the master (slave) to send a multislot packet. In this case, the collision probability is further reduced but the BT throughput decreases as well.

\section{Remarks}

The OLA schemes do not require a centralized controller because they do not perform precise time scheduling of the 802.11 and BT packet traffic. They can either operate as collaborative or noncollaborative coexistence mechanisms and, hence, are able to reduce interference both in the case of collocated and noncollocated devices. If interfering systems other than BT and 802.11 are present, the beneficial effects of the OLA mechanisms still hold as long as BT and 802.11 can estimate the interference pattern of the other systems with sufficient accuracy. Using the OLA scheme at the 802.11 stations can reduce the impact on 


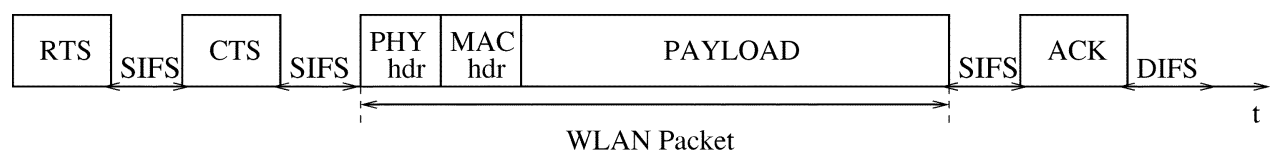

Fig. 3. Timing of a successful IEEE 802.11 packet transmission.

the WLAN performance of any interfering transmission with a periodic pattern. Likewise, the OLA scheme performed by the BT devices is able to counteract the interference generated by any wireless system using a limited frequency span in the ISM band. On the other hand, when interfering devices are collocated in the same physical unit, we expect that collaborative schemes, such as META, give higher throughput than the OLA schemes do.

As already mentioned, we did not consider out-of-band interference. Although adjacent channel interference may lead to an increase of $h_{f}$, it would not affect the OLA schemes. Any frequency channel, where interference is detected, can be simply included in the set of frequencies that BT devices can avoid by using the D-OLA mechanism.

The proposed algorithms have a minor impact on the 802.11 standard and on the BT specification. According to the 802.11 standard, a station shall defer its transmission if it detects a busy channel during the CCA procedure. There are three different CCA modes [3]: 1) a busy channel is reported upon detection of any energy above a certain threshold; 2) a busy channel is reported only upon detection of a DSSS signal, which can be either above or below the energy threshold; and 3) a busy channel is reported upon a DSSS signal with energy above the threshold. Thus, in the V-OLA mechanism, both the PT and the ST modes are compliant with the 802.11 standard and can be implemented by using the appropriate CCA mode among the possible three. The BT specification involves that BT devices dynamically adapt their hopping sequence to the interference conditions by scheduling ACL packets with different length. Thus, the D-OLA scheme exploits a behavior of the BT devices already existing in the specification, and we do not need to change the procedure of hop selection that is performed in the BT hardware. In a piconet, however, the master can only indicate to the slaves the maximum number of slots to use; while, according to the D-OLA mechanism, a slave should interpret the indication from the master as the suggested packet length.

Finally, the D-OLA mechanism is compliant with the FCC regulation Title 47, Part 15.247 (h). ${ }^{2}$ [27] The FCC regulation Part 15.247 (a) states that frequency hopping systems in the 2.4-GHz band shall use 75 hopping frequencies and that the average time of occupancy of any frequency shall not be greater than $0.4 \mathrm{~s}$ within a $30-\mathrm{s}$ period. BT is compliant with this regulation. The D-OLA algorithm does not change the BT hopping pattern; however, since the D-OLA scheme prevents BT from hopping on the 802.11 22-MHz band, it may happen that a set of frequencies less than 75 is actually used, and that the average time of occupancy of any frequency is greater than the above

215.247(h): "The incorporation of intelligence within an FHSS system that permits the system to recognize other users within the spectrum band so that it individually and independently chooses and adapts its hopsets to avoid hopping on occupied channels is permitted. The coordination of frequency hopping systems in any other manner for the express purpose of avoiding the simultaneous occupancy of individual hopping frequencies by multiple transmitters is not permitted." limitation. We notice that the same problem occurs when the $\mathrm{AFH}$ mechanism is used and that the AFH Mode $\mathrm{H}$ has been introduced on purpose to overcome this problem. To make the D-OLA scheme fully compliant with the FCC requirements, a solution similar to the AFH Mode $\mathrm{H}$ could be adopted.

\section{Simulation SCEnARio}

We consider an IEEE 802.11 ad hoc network providing an instantaneous rate equal to $11 \mathrm{Mb} / \mathrm{s}$ and using the DCF MAC scheme. All the stations operate as a self-contained BSS and are able to directly communicate with each other; all stations are assumed to be asynchronous data users with a finite transmission buffer. The arrival of frames from a station's higher layer protocol to the MAC sublayer is modeled with exponential interarrival times and a truncated geometric distribution for the frame lengths [28]. The mean value of the truncated geometric distribution is set to $1500 \mathrm{~B}$, while the maximum frame length is set to the maximum length of the MAC service data unit established by the IEEE 802.11 standard (i.e., 2304 B). The parameter of the exponential distribution is fixed in such a way that the average 802.11 traffic load normalized to the channel capacity is equal to $\lambda_{w}$, a varying parameter in the simulations.

In order to reduce the complexity of the simulation model, the following further assumptions have been introduced.

1) Possible values for the WLAN packet length, if not otherwise specified in the following, have been limited to 500, 1000 , and $1500 \mathrm{~B}$.

2) The request to send/clear to send (RTS/CTS) mechanism is considered always active.

3) No interference is considered from nearby BSSs using the same DSSS spreading sequence.

4) Propagation delay is neglected, which is a reasonable assumption due to the small distance between stations.

5) A two-state Markov model is used to represent the bit error process due to the effect of fading. In state good, the bit error rate is equal to $10^{-10}$; in state $\mathrm{bad}$, it is equal to $10^{-5}$ [28]. The transition probability from good to bad is equal to 0.01 , from bad to good is equal to 0.1 .

An 802.11 transmission is considered to be successful if no collision occurs on the RTS frame and both the data packet and the corresponding acknowledgment sent by the receiver are correctly received. Fig. 3 shows the 802.11 traffic timing in the case of successful packet transmission. If a packet is not correctly received, retransmission will take place according to the backoff procedure defined by the IEEE 802.11 standard. The number of retransmissions before the packet is discarded from the station buffer is limited and set to the Long_Retry_Limit. The values of the IEEE 802.11 parameters used in the simulation model are listed in Table I.

For the BT system, we consider a single piconet where devices are polled by the master on the basis of a round-robin 
TABLE I

PARAMETERS USED IN THE SiMULATION OF THE IEEE 802.11 SySTEM

\begin{tabular}{c|c}
\hline Parameter & Assigned Value \\
\hline Long_Retry_Limit & 10 \\
\hline Physical Header & $144 \mathrm{bits}$ \\
\hline MAC Header & $272 \mathrm{bits}$ \\
\hline Slot_Time & $20 \mu \mathrm{s}$ \\
\hline SIFS & $10 \mu \mathrm{s}$ \\
\hline DIFS & $50 \mu \mathrm{s}$
\end{tabular}

TABLE II

PARAMETERS OF THE BLUETOOTH SYSTEM

\begin{tabular}{c|c}
\hline Parameter & Value \\
\hline$T_{B I}$ & $625 \mu \mathrm{s}$ \\
\hline$T_{B P}$ (1-slot packet) & $366 \mu \mathrm{s}$ \\
\hline$T_{B P}$ (m-slot packet) & $\begin{array}{c}625 \mu \mathrm{s} \text { in slot } i \leq m-1 \\
366 \mu \mathrm{s} \text { in slot } i=m\end{array}$ \\
\hline
\end{tabular}

scheme. Each device has a finite transmission buffer; assumption (4), introduced above for the IEEE 802.11 simulation model, holds also for the BT network. The packet error process over the wireless channel is assumed to be Bernoulli, and the average packet error probability is set to $10^{-3}$. We assume that BT SCO traffic is transmitted by using an HV3-type link, which is expected to be the most popular link type for SCO services [8]. With the HV3-type link for each active connection, a packet is transmitted in both directions every six time slots. In the case of data traffic, a DH1-type link is assumed to be the default operating mode, and therefore, single-slot data packets are used. When the D-OLA scheme is applied, we consider that one- and three-slot packets are used. In the case of three-slot packets, a DH-3 type link is adopted. Notice that in the HV3-, DH1-, and DH3-type link, information in the payload is not FEC encoded [5]. The values of the BT system parameters are reported in Table II.

The arrival of data to a BT device's MAC sublayer is modeled with exponential interarrival times and a truncated geometric distribution for the data unit length. The mean value of the truncated geometric distribution is set to $1500 \mathrm{~B}$ while the maximum data unit length is set to $2800 \mathrm{~B}$, corresponding to the total information carried by $100 \mathrm{DH} 1$ packets. The parameter of the exponential distribution is determined in such a way that the average BT traffic load normalized to the channel capacity is equal to $\lambda_{b}$, a varying parameter in the simulations. Packets that are not correctly received are retransmitted according to the fast automatic repeat request scheme [4], where the sender is notified of the transmission outcome in the first possible slot following the packet transmission.

We model the mutual interference between 802.11 and BT as described in Section III-A and assume a noncollaborative setting. As mentioned in Section III-A, we assume that whenever the transmissions by the BT piconet and the 802.11 stations overlap both in time and in frequency, the signals involved in the collision are received in error. Therefore, we assess the performance of the OLA schemes when the distance between interfering devices is such that the bit error probability due to interference is equal to one. This is a worst-case analysis. We also studied the performance of the proposed schemes in different settings, i.e., by varying the bit error probability experienced by BT and 802.11 when a collision occurs; however, the results that we obtained do not qualitatively differ from those presented below.

\section{PERFormance RESUlts}

Results showing the performance of the OLA mechanisms are derived by using the simulation scenario described in the previous section. The values of the traffic parameters of the BT and 802.11 systems are summarized in Table III.

Fig. 4 presents the 802.11 goodput as a function of the 802.11 traffic load in the case where the BT channel supports one SCO link (left plot) and two SCO links (right plot). Performance of the V-OLA scheme in PT mode and in ST mode are compared with the results obtained in the absence of any coexistence mechanism (indicated in the figure by label N-CM). Goodput is defined as the fraction of transmitted information that is successfully transferred over the radio channel. As expected, the behavior of the 802.11 goodput slightly varies as the WLAN traffic load increases. In contrast, comparing the two plots in Fig. 4, we observe a significant reduction in the 802.11 goodput when we pass from one to two SCO links. However, in the case of one SCO link, by applying the V-OLA PT scheme, we obtain an improvement of $10 \%$ with respect to the case where no coexistence mechanism is implemented. In the case of two SCO links, the improvement is equal to $23 \%$. When the V-OLA ST scheme is used, slightly worse performance than in the case of the V-OLA PT scheme is achieved. In fact, in ST mode, the 802.11 stations do not stop transmitting during the BT busy slots and, thus, the probability to overlap BT SCO packets is higher.

Fig. 5 shows the BT goodput as a function of the 802.11 traffic load for the two V-OLA schemes and in the absence of any coexistence mechanism. The left and the right plots refer to the case where BT supports one and two SCO links, respectively. Clearly, as $\lambda_{w}$ grows, the BT goodput decreases due to the greater interference level. The improvement achieved by using the V-OLA PT scheme can be up to $15 \%$ in the case of one BT SCO link and up to $20 \%$ in the case of two SCO links. In these plots, the gap between the performance obtained through the PT mode and the ST mode is much greater than in Fig. 4, due to the interference caused by unsuccessful RTS and CTS frames. This effect becomes more evident as the 802.11 traffic load grows and the number of BT idle slots decreases, i.e., the collision probability between WLAN stations increases.

Fig. 6 presents the behavior of the 802.11 average packet delay, with the packet delay being the period from the instant a packet is generated to the instant the packet is successfully transmitted. Results are presented as functions of the 802.11 traffic load for the PT and the ST schemes and in the absence of any coexistence mechanism. For very low values of $\lambda_{w}$, the major delay contribution is due to the assumption that 802.11 packets must have a minimum payload equal to $500 \mathrm{~B}$. As $\lambda_{w}$ grows, this effect becomes less relevant, thus, resulting in 
TABLE III

Values of the Traffic Parameters USEd in the Simulation of the OLA Mechanisms

\begin{tabular}{c|c|c|c}
\hline Mechanism & BT Links and Load & 802.11 Load & 802.11 Payload \\
\hline V-OLA (Fig.s 4-6) & 1,2 SCO & $\lambda_{w} \in\{0.1,0.8\}$ & $\{500,1000,1500\}$ bytes \\
\hline D-OLA (Fig.s 7-8) & 2 ACL; $\lambda_{b} \in\{0.1,0.8\}$ & $\lambda_{w}=0.3,0.5$ & $\{500,1000,1500\}$ bytes \\
\hline D-OLA (Fig. 9) & 2 ACL; $\lambda_{b}=0.4$ & $\lambda_{w}=0.3,0.5$ & $200,500,1000,1500$ bytes \\
\hline D-OLA (Fig.s 10-11) & 1 ACL; $\lambda_{b}=0.4$ & $\lambda_{w} \in\{0.1,0.8\}$ & $\{500,1000,1500\}$ bytes \\
\hline
\end{tabular}

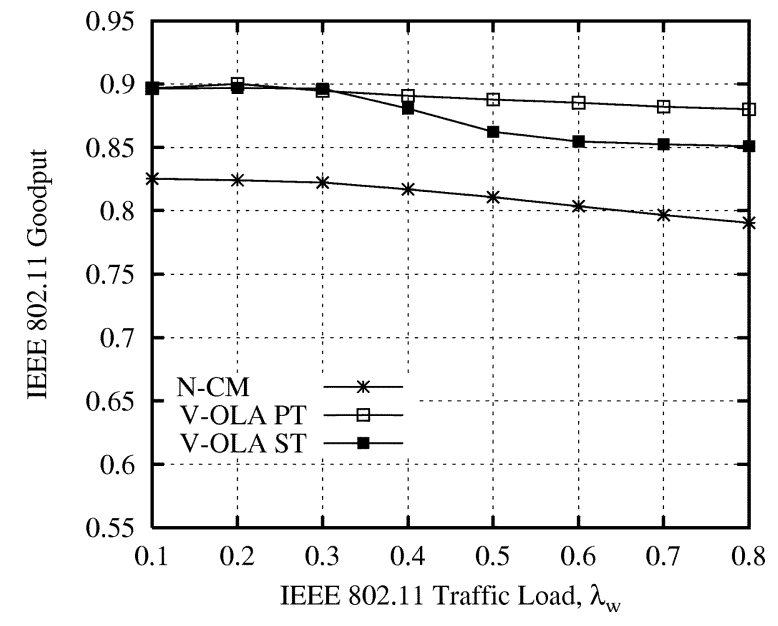

(a)

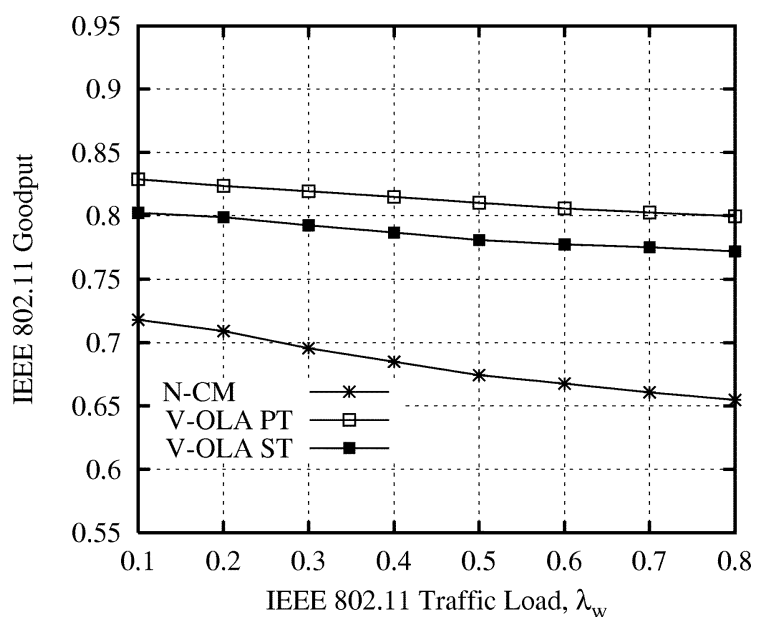

(b)

Fig. 4. (a) IEEE 802.11 goodput when BT supports one SCO link and (b) two SCO links. Performances obtained through the V-OLA schemes and when no coexistence mechanism is applied (N-CM) are compared.

smaller traffic delay. For high values of traffic load, delay is mainly due to collisions between WLAN stations and, in the case of the PT mode, to the lack of BT idle slots. The delay obtained in the case of the ST mode is slightly greater than the delay experienced when no scheme is applied and remains low even when two SCO links are considered. When the PT mode is applied, a low delay is obtained only for one SCO link and $\lambda_{w}$ less than 0.6. When two SCO links are supported and the number of BT idle slots decreases, for almost any value of $\lambda_{w}$, the PT mode gives a delay one order of magnitude higher than in the case of the ST mode.

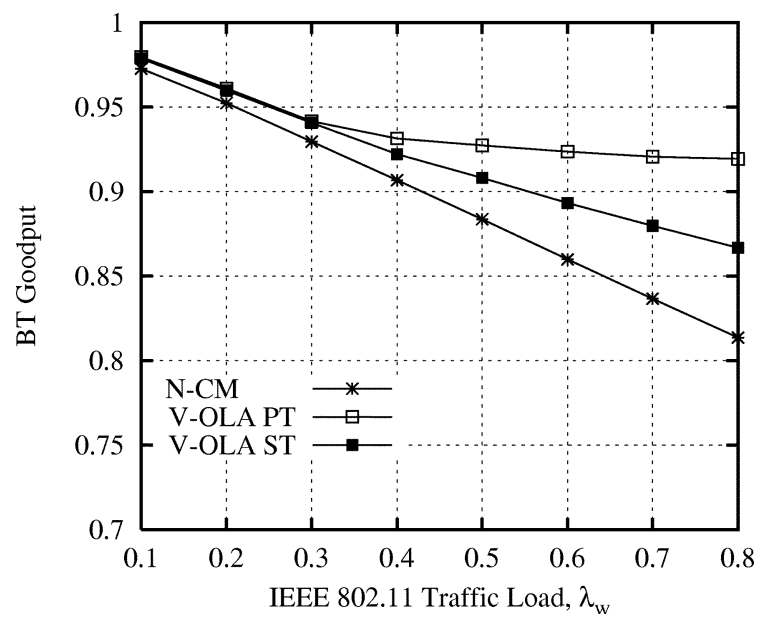

(a)

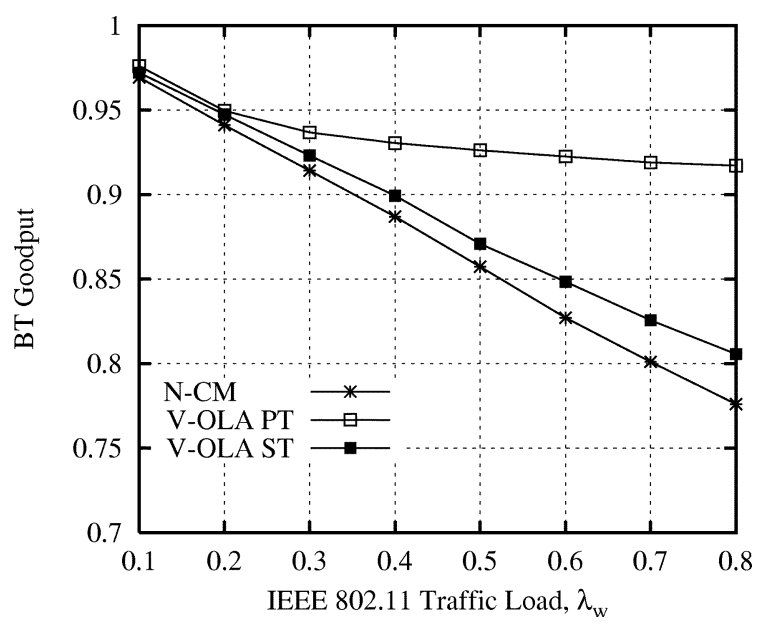

(b)

Fig. 5. (a) BT goodput versus the IEEE 802.11 traffic load when BT supports one SCO link and (b) two SCO links. Performances obtained through the V-OLA schemes and in the case where no coexistence mechanism is applied $(\mathrm{N}-\mathrm{CM})$ are compared.

Figs. 7-9 compare the performance of the D-OLA scheme with the performance obtained in the absence of any coexistence mechanism. Results shown in Figs. 7 and 8 were derived by setting the payload of the 802.11 packets to be equal to 1500 B. The left plot in Fig. 7 presents the 802.11 goodput as a function of the BT traffic load for $\lambda_{w}=0.3$ and 0.5. In the case of the D-OLA scheme, the 802.11 goodput remains almost constant as the BT traffic load increases, but when no scheme is implemented, a significant degradation is observed. The improvement in performance achieved through the proposed coexistence algorithm is as high as $50 \%$ for BT traffic 


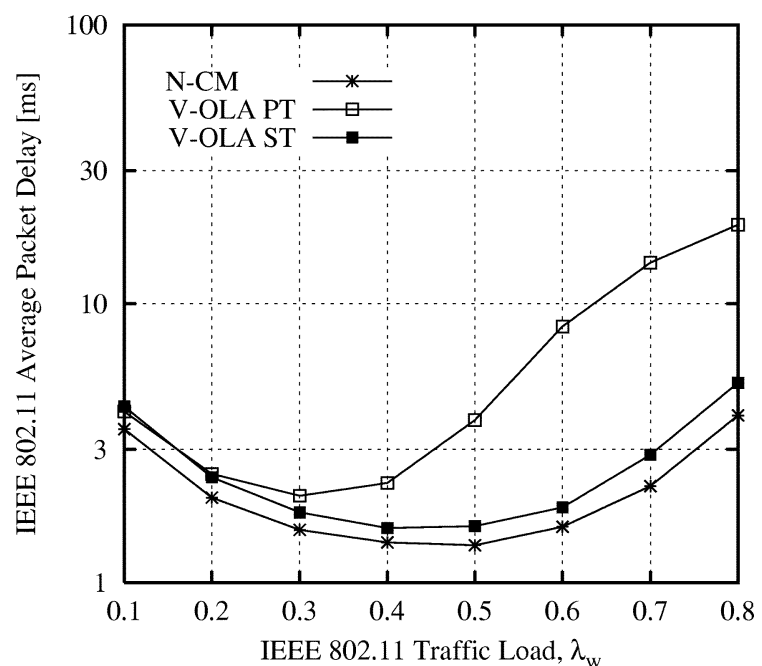

(a)

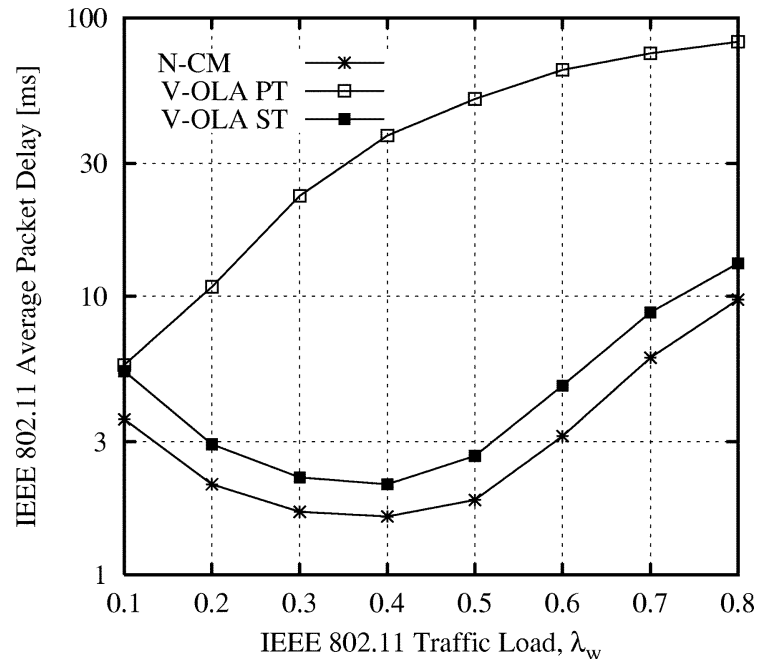

(b)

Fig. 6. (a) IEEE 802.11 average packet delay versus traffic load $\lambda_{w}$ in the presence of one BT SCO link and (b) two BT SCO links. Performances obtained through the V-OLA schemes and in the case where no coexistence mechanism is applied (N-CM) are compared.

load equal to 0.8 . As expected, results slightly change as the 802.11 traffic load varies.

Similar considerations hold for the results presented in the right plot in Fig. 7 where the BT goodput is shown as a function of $\lambda_{w}$ and for different values of the BT traffic load. In this case, the improvement in performance obtained through the D-OLA scheme is equal to $24 \%$ for $\lambda_{w}=0.8$.

Fig. 8 shows the BT average packet delay versus the BT traffic load for $\lambda_{w}=0.3$ and 0.5 . For $\lambda_{w}=0.5$, the delay experienced when the D-OLA algorithm is applied is slightly higher than the delay achieved in the absence of any coexistence mechanism; for $\lambda_{w}=0.3$, the two curves overlap. This shows that the D-OLA scheme greatly mitigates the mutual interference between 802.11 and BT without causing a reduction in the BT throughput.

Fig. 9 presents the 802.11 goodput as the payload of the 802.11 packet varies for $\lambda_{w}=0.3$ and 0.5 . Results were derived from simulations where the 802.11 payload was fixed to a constant value. The plot confirms the improvement
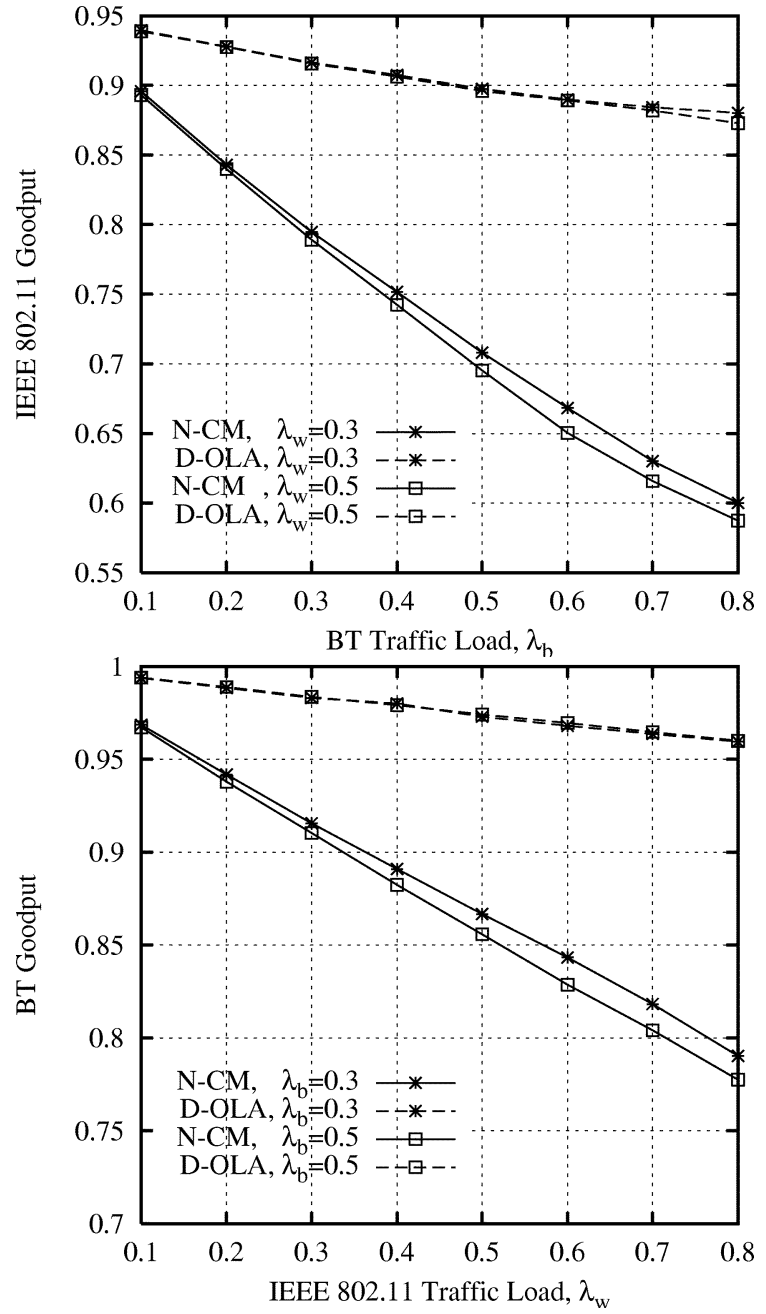

Fig. 7. Goodput of the IEEE 802.11 and the BT system in the presence of BT data links. Performances obtained through the D-OLA scheme and when no coexistence mechanism is applied $(\mathrm{N}-\mathrm{CM})$ are compared.

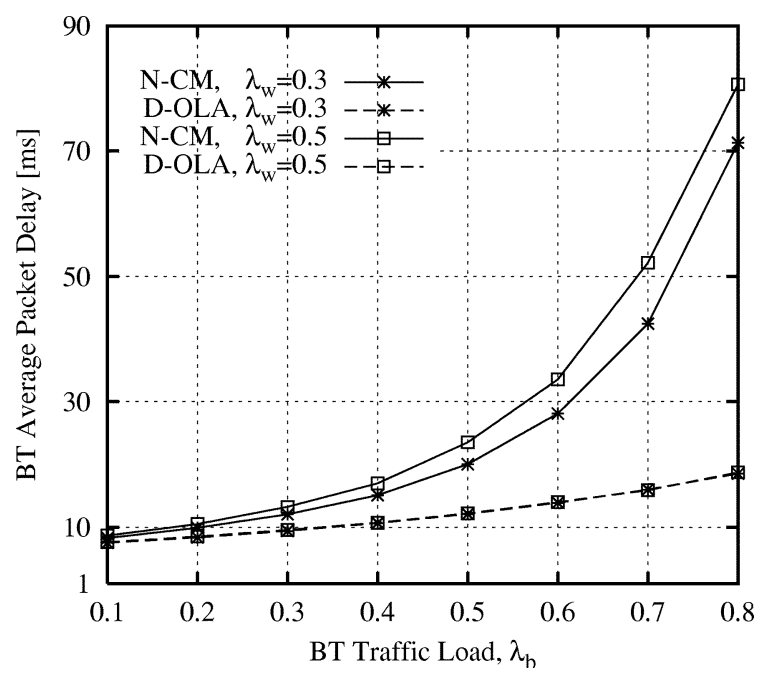

Fig. 8. BT average packet delay versus BT traffic load in the presence of BT data links. Performances obtained through the D-OLA scheme and when no coexistence mechanism is applied (N-CM) are compared for different values of the IEEE 802.11 traffic load.

achieved through the D-OLA algorithm. As expected, when no coexistence mechanism is used, lower values of WLAN 


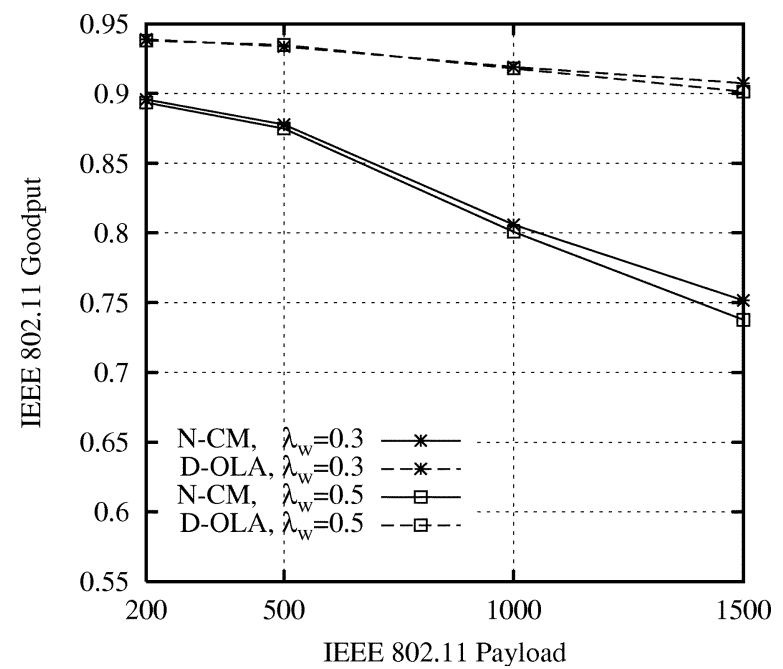

Fig. 9. IEEE 802.11 goodput versus the 802.11 packet payload in the presence of BT data links and BT traffic load equal to 0.4. Performances obtained through the D-OLA scheme and when no coexistence mechanism is applied (N-CM) are compared for different values of the IEEE 802.11 traffic load.

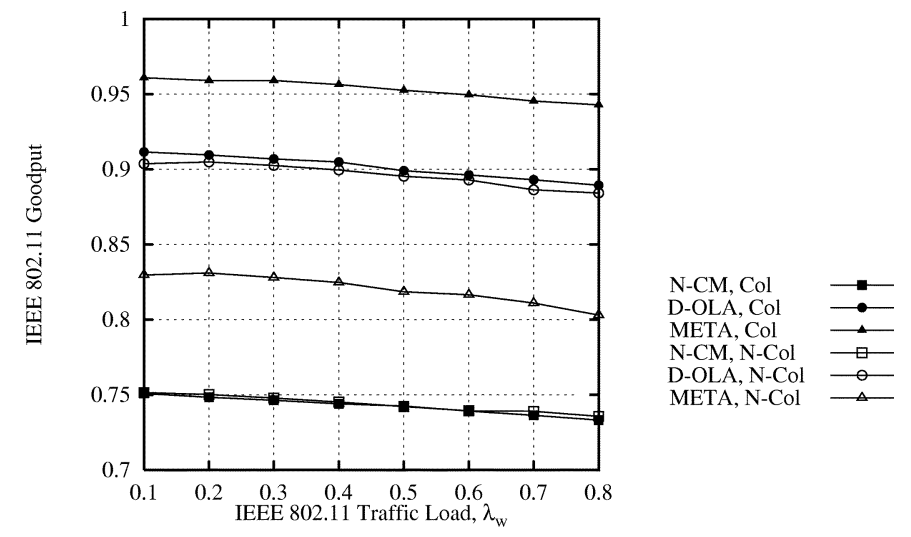

Fig. 10. IEEE 802.11 goodput in presence of one interfering BT device supporting data traffic. Performances obtained through the D-OLA algorithm, the META scheme, and in the case where no coexistence mechanism is applied (N-CM) are compared. Results obtained in the scenario with and without collocated interfering devices are indicated by labels Col and N-Col, respectively.

payload give higher 802.11 goodput because the packet error probability decreases. It is interesting to notice that in the case of the D-OLA scheme, the WLAN payload has a negligible impact on the performance.

Finally, we present results showing that collaborative schemes, such as META [11], outperform the OLA mechanisms when interfering devices are collocated in the same physical unit. The opposite is true when noncollocated devices interfere with each other.

Fig. 10 presents the 802.11 goodput achieved through the D-OLA algorithm and through the META scheme compared with the performance obtained when no coexistence mechanism is applied. The plot is obtained by assuming a BT data link with normalized traffic load equal to 0.4 and setting the WLAN payload to be equal to $1500 \mathrm{~B}$. Two different scenarios are studied: 1) a BT device interferes with one collocated 802.11 device only and 2) a BT device interferes with one collocated

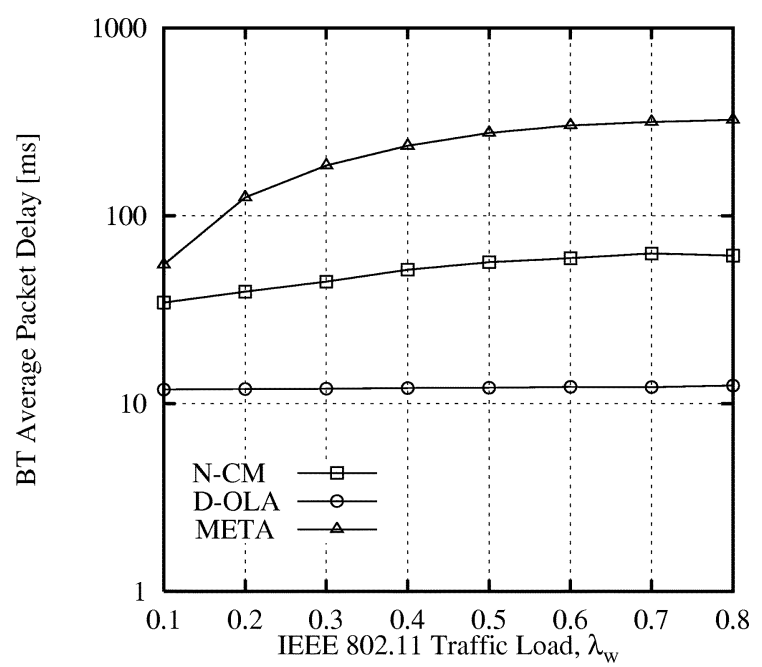

Fig. 11. BT average packet delay when one IEEE 802.11 and one BT collocated devices are considered. The BT device supports data traffic. Performances obtained through the D-OLA algorithm, the META scheme and, when no coexistence mechanism is applied (N-CM) are compared.

and other noncollocated 802.11 devices. Clearly, in the first scenario, META gives the best performance. However, the META goodput greatly decreases as noncollocated 802.11 devices are considered while the performance of the D-OLA algorithm is almost unchanged.

Fig. 11 presents the average packet delay for a BT device. The curves in the plot refer to the case where only two collocated devices interfere; results do not significantly vary as noncollocated devices are considered. When META is applied, the 802.11 traffic load significantly affects the BT packet delay. When the D-OLA algorithm is adopted, the packet delay is constant as $\lambda_{w}$ increases. Also, by using META, we obtain a BT packet delay that is one order of magnitude greater than in the case of the D-OLA scheme.

\section{IMPACT OF MiCROWAVE OVEN INTERFERENCE ON IEEE 802.11 PERFORMANCE}

Microwave ovens operate in the ISM bands and are largely used in residential and commercial environments. As shown in [29]-[32], the effect of the microwave ovens interference on the bit-error rate of WLANs operating at $2.4 \mathrm{GHz}$ is quite significant.

We consider residential transformer-type microwave ovens, which include a single magnetron tube and whose power consumption is roughly equal to $600 \mathrm{~W}$. Typically, they are active for a time period of about $8 \mathrm{~ms}$ over a power cycle of $20 \mathrm{~ms}$ when the power supply frequency is equal to $50 \mathrm{~Hz}$ or of $16 \mathrm{~ms}$ when the power supply frequency is equal to $60 \mathrm{~Hz}$. Since the microwave oven interference has a periodic pattern, we can apply the V-OLA schemes and study their ability to reduce the impact of the microwave oven interference on the 802.11 performance. The following two scenarios are considered [29], [30], [32]: 1) the microwave oven is located $3 \mathrm{~m}$ away from the 802.11 receiver causing a bit error probability equal to 0.01 and 2) the microwave oven is located $0.5 \mathrm{~m}$ away from the 802.11 device resulting in a bit error probability equal to one. 


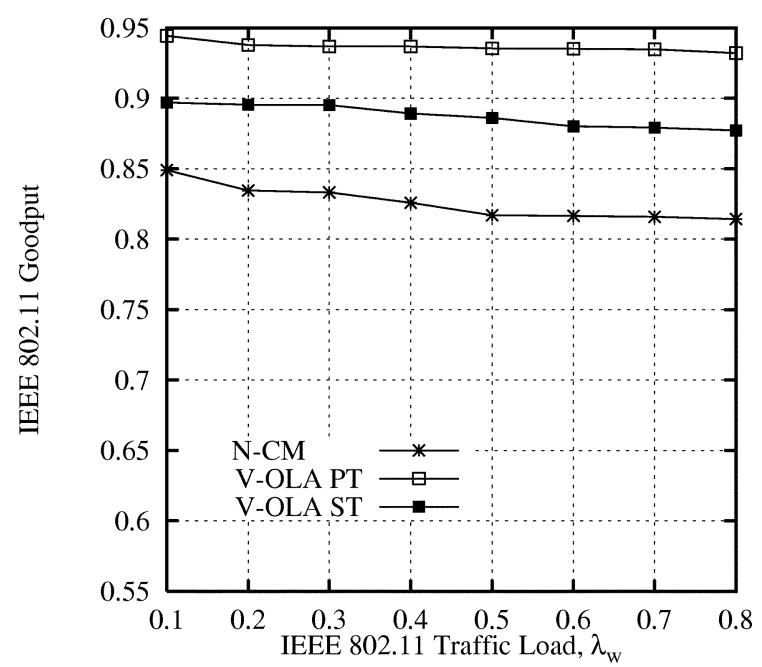

(a)

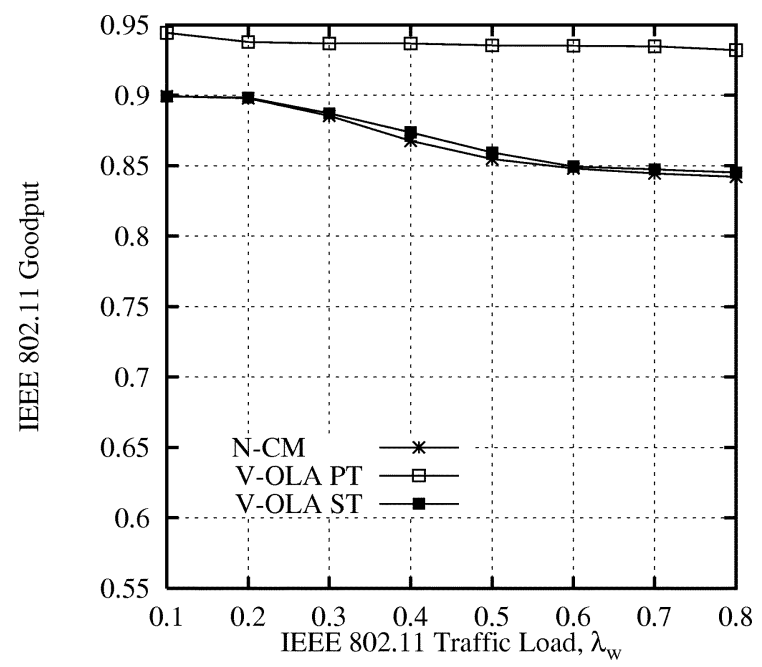

(b)

Fig. 12. IEEE 802.11 goodput in presence of the interference generated by a microwave oven. (a) The distance between the microwave oven and the 802.11 receiver is roughly equal to 3 and (b) $0.5 \mathrm{~m}$. Performances obtained through the $\mathrm{V}$-OLA schemes and when no coexistence mechanism is applied (N-CM) are compared.

Fig. 12 illustrates the 802.11 goodput obtained through the V-OLA algorithms with that achieved when no coexistence mechanism is applied. Results in the left and right plots are derived by assuming the 802.11 bit-error probability to be equal to 0.01 and 1.0 , respectively; the power cycle of the microwave oven is assumed to be equal to $20 \mathrm{~ms}$. In the first scenario, both the V-OLA schemes are able to mitigate the microwave oven interference. In the second scenario, two effects take place: 1) the 802.11 goodput is higher than in the first scenario and 2) an improvement in performance is obtained only when the VOLA PT scheme is applied. This is because the WLAN uses the RTS/CTS access protocol and errors in the reception of either the RTS or the CTS message prevent the 802.11 stations from transmitting. Therefore, while the microwave oven is active (an erroneous reception of the handshaking messages occurs with probability equal to one), no packet is transmitted over the 802.11 channel, and the performance of the WLAN in absence of any coexistence mechanism is the same as that obtained through the V-OLA ST scheme.

\section{CONCLUSION}

In this paper, the problem of mutual interference between different wireless technologies operating in the 2.4-GHz ISM bands was addressed. We considered IEEE 802.11 WLANs and BT-based WPANs. Two different coexistence mechanisms based on traffic scheduling techniques were proposed: one to be applied at the WLAN stations to avoid overlap between 802.11 traffic and BT voice packets and the other to be executed at the BT devices to avoid overlap in frequency between 802.11 traffic and BT data packets.

The main advantages of the proposed mechanisms are as follows.

1) They do not require a centralized traffic scheduler.

2) They can be implemented either when 802.11 and BT are able to exchange information (collaborative coexistence mechanism) or when they acquire this information by detecting interfering transmissions over the radio channel (noncollaborative coexistence mechanism).

3) They are able to mitigate interference between collocated and noncollocated BT and 802.11 devices.

4) They have a minor impact on the IEEE 802.11 standard and the BT specification.

Results indicate that the proposed mechanisms reduce the interference between 802.11 and BT, outperforming collaborative mechanisms when noncollocated devices interfere with each other. Results showing the ability of the proposed schemes to cope with interference caused by microwave ovens also were presented.

\section{REFERENCES}

[1] J. M. Peha, "Wireless communications and coexistence for smart environments," IEEE Pers. Commun., vol. 7, pp. 66-68, Oct. 2000.

[2] B. P. Crow, I. Widjaja, J. G. Kim, and P. T. Sakai, "IEEE 802.11 wireless local area networks," IEEE Commun. Mag., vol. 35, pp. 116-126, Sept. 1997.

[3] Local and Metropolitan Area Networks: Wireless LAN, ANSI/IEEE Standard 802.11.

[4] J. C. Haartsen, "The bluetooth radio system," IEEE Pers. Commun. Mag., vol. 7, pp. 28-36, Feb. 2000.

[5] Bluetooth Core Specification [Online]. Available: http://www.bluetooth.com

[6] IEEE 802.15 WPAN Task Group 2 (TG2) [Online]. Available: http://www.ieee802.org/15/pub/TG2.html

[7] WiFi (802.11b) and Bluetooth: An Examination of Coexistence Approaches (2001, Mar.). [Online]. Available: http://www.mobilian.com/ whitepaper_frame.htm

[8] S. Shellhammer. (2001, Mar.) SCORT-An Alternative to the Bluetooth SCO Link for Operation in an Interference Environment 01/145r 1 IEEE 802.15-01/145r1. [Online]. Available: http://www.ieee802.org/15/ pub/TG2-Coexistence-Mechanisms.html

[9] D. Cypher. (2000, Mar.) Coexistence, Interoperability, and Other Terms IEEE 802.15-99/134r1. [Online]. Available: http://www.ieee802.org/15/ pub/TG2-Technical-Presentations.html

[10] V. Arunachalam and B. Treister. (2001, July) Clause 5.3-Overview of Coexistence Mechanisms. IEEE P802.15 Working Group for WPANs. [Online]. Available: http://www.ieee802.org/15/pub/TG2-Draft.html

[11] J. Lansford and A. P. Stephens. (2001, July) TG2 Mobilian Draft Text. IEEE P802.15 Working Group for WPANs IEEE 802.15-01/300r1. [Online]. Available: http://www.ieee802.org/15/pub/TG2-Draft.html

[12] S. Shellhammer. (2001, Jan.) Collocated Collaborative Coexistence Mechanism: TDMA of 802.11 and Bluetooth IEEE 802.15-01/025r0. [Online]. Available: http://www.ieee802.org/15/pub/TG2-CoexistenceMechanisms.html

[13] — , (2001, July) IEEE 802.15 Clause 14.1-Collaborative Co-Existence Mechanism IEEE 802.15-01/340r0. IEEE P802.15 Working Group for WPANs. [Online]. Available: http://www.ieee802.org/15/ pub/TG2-Draft.html 
[14] R. E. Van Dyck and A. Soltanian. (2001, July) IEEE 802.15 Clause 14.1-Collaborative Co-Located Coexistence Mechanism. IEEE P802.15 Working Group for WPANs IEEE 802.15-01/364r0. [Online]. Available: http://www.ieee802.org/15/pub/TG2-Draft.html

[15] H. Gan and B. Treister. (2000, Nov.) Adaptive Frequency Hopping Implementation Proposals for IEEE 802.15.1/2 WPAN IEEE 802.1500/367r0. [Online]. Available: http://www.ieee802.org/15/pub/TG2-Coexistence-Mechanisms.html

[16] B. Treister et al.. (2001, July) IEEE 802.15 Clause 14.3-Adaptive Frequency Hopping IEEE 802.15-01/366r1. IEEE P802.15 Working Group for WPANs. [Online]. Available: http://www.ieee802.org/15/pub/TG2Draft.html

[17] B. Treister, H. B. Gan, K. C. Chen, H. K. Chen, A. Batra, and O. Eliezer. (2001, May) Components of the AFH Mechanism IEEE 802.1501/252r0. [Online]. Available: http://www.ieee802.org/15/pub/TG2-Coexistence-Mechanisms.html

[18] N. Golmie. (2001, July) Non-Collaborative MAC Mechanisms IEEE 802.15-01/316r0. IEEE P802.15 Working Group for WPANs. [Online]. Available: http://www.ieee802.org/15/pub/TG2-Draft.html

[19] M. B. Shoemake. (2001, Jan.) Proposal for Power Control for Enhanced Coexistence IEEE 802.15-00/081r0. [Online]. Available: http://www.ieee802.org/15/pub/TG2-Coexistence-Mechanisms.html

[20] A. Kamerman, "Spread spectrum schemes for microwave frequency WLANs," Microw. J., vol. 40, no. 2, pp. 80-90, Feb. 1997.

[21] — "Coexistence Between Bluetooth and IEEE 802.11 CCK Solutions to Avoid Mutual Interference," IEEE, 802.11-00/162, July 2000.

[22] S. Shellhammer, "Packet Error Rate of an IEEE 802.11 WLAN in the Presence of Bluetooth," IEEE, 802.15-00/133r0, May 2000.

[23] D. C. Johnson, "Interference Potential of Wideband Frequency Hopping Systems on Packet Data Systems," IEEE, 802.11-99/205, Sept. 1999.

[24] J.Zyren. (1999, June) Reliability of IEEE 802.11 Hi rate DSSS WLANs in high density bluetooth environment. Bluetooth'99 [Online] Available: http://citeseer.nj.nec.com/336567.html

[25] I. Howitt, "WLAN and WPAN coexistence in UL band," IEEE Trans. Veh. Technol., vol. 50, pp. 1114-1124, July 2001.

[26] C. F. Chiasserini and R. R. Rao, "Performance of IEEE 802.11 WLAN in a bluetooth environment," presented at the IEEE Wireless Communications Networking Conf., Chicago, IL, Sept. 2000

[27] "Radio Frequency Devices," Federal Communications Commission, US Government Printing Office, Code of Federal Regulations, Title 47, Part 15, Sub Part C, Section 247, Oct. 2000.

[28] B. P. Crow, I. Widjaja, J. G. Kim, and P. Sakai, "Investigation of the IEEE 802.11 medium access control (MAC) sublayer functions," in Proc. INFOCOM'97, Mar. 1997, pp. 126-133.

[29] S. Miyamoto, Y. Yamanaka, and T. Shinozuka, "A study of the effect of microwave oven interference on the performance of digital radio communications systems," Electron. Commun. Japan, vol. 80, no. 12, pp. $58-67,1997$.

[30] S. Miyamoto and N. Morinaga, "Effect of microwave oven interference on the performance of digital radio communications systems," in Proc. IEEE ICC, Montreal, Canada, June 1997, pp. 51-55.
[31] A. Kamerman and N. Erkocevic, "Microwave oven interference on wireless LANs operating in the 2.4-GHz ISM band," in Proc. 8th IEEE Int. Symp. Personal, Indoor and Mobile Radio Communications, Helsinki, Finland, Sept. 1997, pp. 1221-1227.

[32] S.-T. Sheu, Y.-H. Lee, M.-H. Chen, Y.-C. Yu, and Y.-C. Huang, "PLFC: The packet length fuzzy controller to improve the performance of WLAN under the interference of microwave oven," in Proc. IEEE Globecom, San Francisco, CA, Nov. 2000, pp. 1427-1431.

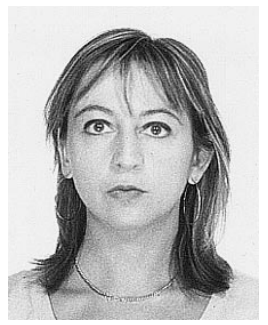

Carla-Fabiana Chiasserini (S'98-M'00) received the electrical engineering degree (summa cum laude) from the University of Florence, Florence, Italy, in 1996 and the Ph.D. degree from the Politecnico di Torino, Torino, Italy, in 1999.

Since 1999, she has been with the Department of Electrical Engineering at Politecnico di Torino, where she is currently an Assistant Professor. She was at the Center for Wireless Communications, University of California, San Diego, CA, as a Visiting Researcher from 1999 to 2001. Her research interests include architectures, protocols, and performance analysis of wireless networks for integrated multimedia services.

Ramesh R. Rao (M'85-SM'90) received the Bachelor's degree in electrical and electronics engineering from the University of Madras, Madras, India, in 1980, and the M.S. and Ph.D. degrees from the University of Maryland, College Park, in 1982 and 1984, respectively.

Since 1984, he has been on the faculty of the Department of Electrical and Computer Engineering at the University of California, San Diego (UCSD), where he is currently Professor and Director of the San Diego Division of the California Institute of Telecommunications and Information Technology. Prior to this appointment, he served as the Director of the UCSD Center for Wireless Communications and was the Vice Chair of Instructional Affairs in the Department of Electrical and Computer Engineering. His research interests include architectures, protocols, and performance analysis of wireless, wireline, and photonic networks for integrated multimedia services. He served as the Editor of the INFORMATION THEORY SOCIETY NEWSLETTER from 1993 to 1995 and is the founding Web Editor of the Information Theory Society web site.

Dr. Rao was elected to the IEEE Information Theory Society Board of Governors (from 1997 to 1999 and 2000 to 2002). He is the Editor for Packet Multiple Access of the IEEE TRANSACTIONS ON COMMUNICATIONS and is a member of the Editorial Board of the ACM/BALTZER WIRELESS NETWORK JOURNAL, as well as IEEE Network Magazine. 